\title{
Análisis de recursos virtuales sobre documentación musical
}

\author{
María F. Sánchez Hernández \\ Universidad Rey Juan Carlos, Madrid (España)
}

\section{Resumen}

Se presentan y analizan los recursos electrónicos más importantes sobre documentación musical, dividiéndolos en cuatro grandes grupos: fuentes de información: glosarios, guías, bases de datos, revistas, buscadores, recursos musicales, etcétera; unidades de información: centros de documentación, archivos y bibliotecas (tanto de ámbito público como privado); universidades donde se imparten estudios superiores en música; y derecho de autor y propiedad intelectual. La búsqueda inicial se contrastó con documentalistas de diferentes unidades de información. Los resultados obtenidos en este trabajo sirven de base para futuros estudios, así como de guía para profesionales de la documentación sonora y de otras unidades de información especializadas en música, pues facilitan tanto un acercamiento a este mundo como un conocimiento de los recursos y fuentes de información musicales disponibles en Internet.

Palabras clave: Documentación musical. Internet. Fuentes de información. Recursos electrónicos. Unidades de Información.

\begin{abstract}
The main electronic resources on musical documentation are described and analysed. They were collected through Internet searches and by interviews with music documentalists. The resources were classified into four groups: information sources (glossaries, data bases, guides, magazines, search engines, musical resources...); information units: documentation centers, archives and libraries (public as much as private area); universities where superior studies in music are imparted; and author rights and intellectual property. The results of this work will hopeful serve as a background for future studies, as well as a guide for the soundrelated information professionals and librarians in music information units, and, of course, for user reference.
\end{abstract}

Key words: Musical documentation. Internet. Information sources. Electronic resources. Information units. 


\section{Introducción}

La cultura musical ha proliferado de un modo rápido. En nuestros hogares contamos con equipos de alta fidelidad, con reproductores de CD, de DVD, etcétera. Las emisoras de radio cuentan con una importante presencia de programas dedicados a música de todos los estilos, y ofrecen la posibilidad de escucharlos a través de Internet. La televisión dedica canales exclusivamente a programas musicales o de videoclips. En los programas educativos y lúdicos se incorpora la música no solo para distracción y deleite, sino como disciplina académica. Incluso se utiliza como terapia con las técnicas conocidas como musicoterapia.

Los documentos musicales son aquellos "cuyo contenido es música", independientemente de sus soportes — casetes, discos de vinilo, CD, partituras musicales, archivos MIDI, MP3, revistas, carteles, etcétera- Desde los soportes tradicionales para la conservación de la documentación musical, como es el papel, a los de última generación electrónicos se ha evolucionado notablemente con la llegada de las nuevas tecnologías y sus avances, lo que favorece tanto el acceso a ellos como su consulta virtual.

Las partituras musicales conservadas en archivos con formato TIF o JPG, entre otros, se difunden a través de Internet o en soportes ópticos gracias a las técnicas de digitalización empleadas. Actualmente podemos localizar la partitura que más nos interese en la Red e imprimirla, o bien escucharla en distintos soportes, así como conseguir la traducción de un aria de ópera.

El derecho de autor o derecho a la propiedad intelectual no nace con la sociedad actual: ya en el año 25 a. C. Marco Vitruvio lo recogía en su Libro Séptimo de Architectura. A través de Internet también nos podemos informar de estos derechos, así como de las normativas y convenios reconocidos por instituciones, fundaciones, asociaciones de autores o por la Administración Pública.

\section{Análisis de los recursos electrónicos}

\subsection{Glosarios}

Glosario del jazz

http://www.apoloybaco.com/GlosariodeJazz_r.htm (2007-03-17)

Se trata de un completo glosario dedicado al jazz. Está ordenado alfabéticamente y mediante sus enlaces podemos acceder de un modo rápido y eficaz al término que nos interese.

\section{Himnario digital}

http://himnariodigital.org/glossary_a-1.html (2006-07-03)

En esta URL encontraremos una colección de archivos MIDI, letras y notas históricas acerca de los autores y compositores de los himnos en castellano e inglés. 
El enlace al glosario es una completa herramienta para profesionales o interesados por la música.

\subsection{Guías y directorios}

Guía profesional de la música

http://www.musices.com/ (2006-07-03)

Esta guía interactiva ofrece información de conciertos, músicos de sesión, editoriales y librerías especializadas en música, directorios especializados (de colectivos, asociaciones, clubs de fans, conservatorios, escuelas de música, artistas internacionales, bandas de música, etcétera), así como enlaces dedicados a agencias de publicidad y de espectáculos.

\section{Music Resources}

http://www.siba.fi/Kulttuuripalvelut/music.html (2006-07-03)

Creada por la Sibelius Academy (Finlandia), que está especializada en enseñanza musical, presenta sitios webs, catálogos de música, bibliotecas musicales, música religiosa, publicaciones on-line, composiciones, archivos MIDI, etcétera.

Rock on the Net

http://www.rockonthenet.com/ (2006-07-03)

Se trata de una guía especializada en música rock con información de conciertos, referencias de grupos históricos, foros, etcétera.

\section{Guiamusical.com}

http://www.guiamusical.com (2006-07-03)

Permite realizar búsquedas por materias, comunidades o géneros musicales. Entre sus servicios destacan los de buscador/enlaces, tablón de anuncios, encuestas, noticias y agenda de conciertos, entre otros.

\section{Naciones Unidas}

http://www.unesco.org/cgi-bin/webworld/portal_bib2/cgi/page.cgi?g= Libraries\%2FCulture\%2FArts\%2FMusic\%2Findex.shtml;d=1 (2006-07-03)

Directorio donde se recopila bibliotecas especializadas en música, con un total de 78 europeas y 35 americanas ordenadas por países, y se ofrece un resumen de cada una de ellas, así como su localización en Internet.

Music homepages in the world http://www.cilea.it/music/musworld.htm (2006-07-03)

Relación de las páginas webs más importantes sobre música agrupadas por países, aunque también pueden ser consultadas por temas. Aporta enlaces de música, televisión y radio, además de información sobre géneros musicales, compositores, 
cantantes, instrumentos y publicaciones. También conecta con un buscador especializado.

Centre Pompidou. IRCAM

http://mediatheque.ircam.fr/internet/note.html (2006-07-03)

Es una mediateca virtual en cuyo completo repertorio podemos encontrar enlaces con páginas webs relacionadas con el mundo de la música y agrupadas en bases de datos, organismos, bibliotecas, foros públicos, publicaciones periódicas e información en Internet.

Windowsmedia

http://windowsmedia.msn.com/radiotuner/MyRadio.asp (2006-07-03)

Entre las guías de radio en Internet destacamos esta, que tiene enlaces predeterminados para escuchar la radio (BBC Español, Emisión Digital...) a través de Internet. Además contiene un buscador para localizar emisoras especializadas en jazz, rock, noticias y coloquios, listas de éxitos, etcétera.

\subsection{Buscadores}

The Italian Music Homepage

http://www.cilea.it/music/mushhoms.htm (2006-07-03)

Ofrece un sistema de búsqueda exclusivamente dedicado a la música organizado por países y tiene múltiples enlaces a organizaciones y sociedades musicales, publicaciones, géneros, bases de datos, bibliotecas, agencias, sitios webs, agenda, archivos musicales, etcétera.

\section{Music Search}

http://www.musicsearch.com (2006-07-03)

Es un motor de búsqueda con una base de datos de más de 20000 direcciones de páginas webs musicales. Su consulta se puede realizar mediante una búsqueda simple a través de descriptores y categorías temáticas.

La mà de guido

http://www.webcom.com/musics/ (2006-07-03)

Es una editorial de música impresa que incluye un sistema de búsqueda para realizar consultas y ofrece la posibilidad de enviar información para completar su base de datos. También contiene recursos sobre música clásica en España, y especialmente en Cataluña, con referencias bibliográficas relativas a compositores e intérpretes de todos los tiempos. 


\subsection{Bases de datos}

RISM On Line: International Inventory of Musical Sources

http://rism.ub.uni-frankfurt.de (2006-07-03)

Podemos acceder a cuatro bases de datos: RISM Series AII Music Mss., con manuscritos musicales y registros bibliográficos fechados entre 1600 y 1850; RISM US Libretto Database, que contiene 11000 registros bibliográficos de libretos y música escénica, desde los inicios de este género hasta inicios del siglo XIX; RISM Libraries Directory, directorio de bibliotecas musicales de todo el mundo; y RISM Bibliographic Citations Database, con referencias bibliográficas sobre los catálogos temáticos y fuentes secundarias recogidas en el RISM AII y en RISM US Libretto Database.

International Index to Music Periodicals (IIMP)

http://music.chadwyck.com (2006-07-03)

Se trata de una gran base de datos con índices de 430 revistas musicales de más de 20 países.

\section{Gracenote}

http://www.cddb.com (2006-07-03)

Es una base de datos discográfica que recopila distintos géneros de música: clásica, pop, flamenco, ópera, etcétera. Se puede realizar búsquedas por artistas y por títulos de álbumes y/o canciones.

International Database of the Choral Music

http://musica.u-strasbg.fr (2006-07-03)

Es la mayor base de datos dedicada a la música coral, con más de 68000 fichas. Promovida por la Federación Internacional de Música Coral, la consultan entre 50 y 80 usuarios diariamente mediante búsquedas simples o completas búsquedas avanzadas.

\section{Music Education Search System}

http://www.music.miami.edu/mess/ (2006-07-03)

Mantenida por Edward P. Asmus, ofrece bases de datos de importantes revistas, como Journal of Research in Music Education, Journal of Music Teacher Education, Music Education, Philosophy of Music Education Review, Journal of Music Education, Quarterly Journal of Music Teaching and Learning... Una de sus bases de datos tiene 13000 entradas, y otra, de abstracts, 4500.

\section{ProMusicFind.com}

http://www.promusicfind.com/new/aboutus.shtml (2006-07-03)

Base de datos dedicada a festivales de música, artículos, asociaciones, artistas, publicaciones, búsquedas, guías, etcétera.

Scire. $13: 2$ (jul.-dic. 2007) 167-184. ISSN 1135-3716. 


\section{Gramophone}

http://www.gramophone.co.uk/ (2006-07-03)

Ofrece una base de datos de revistas de música clásica.

Classical Net

http://www.classical.net/ (2006-07-03)

Tiene 6000 archivos a los que se accede por medio de 4000 enlaces dedicados a la música clásica; es una gran base de datos de compositores en la que se destacan sus trabajos, publicaciones, etcétera.

Harmonie Park Press

http://www.harmonieparkpress.com/ (2006-07-03)

Contiene índices desde 1948 de más de 100 títulos de publicaciones periódicas y libros especializados en música. En sus bases de datos nos ofrece referencias bibliográficas on-line con información sobre autor, título, resumen, número de páginas, ISBN, precio y año de cada una de las obras incluidas.

\section{Music Database Team}

http://www.fmpweb.hsd.uvic.ca/merb/ (2006-07-03)

Se trata de una base de datos bibliográfica con 31000 referencias de 35 publicaciones periódicas dedicadas a la música y a la educación musical desde 1956 hasta la actualidad, completamente indizadas por títulos, autores y materias.

Center for the History of Music Theory and Literature http://www.music.indiana.edu/tml/start.html (2006-07-03)

Página que contiene un tesauro de música medieval latina de la Edad Media y del Renacimiento, además de permitir utilizar sus bases de datos.

\subsection{Recursos dedicados a música clásica}

\section{Kareol}

http://www.supercable.es/ ealmagro/kareol/index.htm (2006-07-03)

Está orientado fundamentalmente al mundo de la ópera, pero también contiene oratorios, cantatas, corales y todas aquellas obras de música clásica en las que interviene la voz humana. Todos los textos de la página son bilingües; se traducen al castellano óperas, cantatas, etcétera. Podemos realizar búsquedas por autores, por obras, por colaboradores y por obras en edición.

\section{ArkivMusic}

http://www.classical.net/music/links/operalnk.html (2006-07-03)

Se trata de una gran herramienta electrónica para la música clásica, donde podemos localizar libros y librerías especializadas, revistas, foros de debate, un di- 
rectorio de los principales teatros del mundo y un largo etcétera a través de los más de 100 enlaces que ofrece.

La Ópera

http://usuarios.lycos.es/Papageno/portada.htm (2006-07-03)

Es una página dedicada a la ópera donde encontramos secciones con información de los principales teatros líricos, un índice de cantantes clasificados por voces, un diccionario de óperas, compositores, libretistas y argumentos, etcétera.

Directory of Music User Guides for Libraries

http://www.library.yale.edu/ segglstn/mugdir/index.htm (2006-07-03)

Confeccionado por el Bibliographic Instruction Subcommittee de la Music Library Association, es uno de los más completos directorios-guía sobre música y uno de los mejores repertorios existentes sobre música en línea. Está compuesto por ocho secciones sobre bibliografías, recursos electrónicos, fuentes de interés para la educación musical, publicaciones periódicas, catálogos en línea de bibliotecas musicales, etcétera.

\subsection{Revistas}

\section{Orfeo Ediciones}

http://www.orfeoed.com/ (2006-07-03)

Es una editorial fundada en 1996 que publica libros y revistas de esta temática. Algunas de las cabeceras y títulos más conocidos son Melómano (desde 1996, dedicada a la música clásica), Melómano Danza y Más Jazz (desde 1998). Entre sus ediciones destaca el Libro de oro de la música en España. Se trata de la historia de las instituciones musicales españolas contada año por año, en sus diez volúmenes publicados desde 1996 hasta la actualidad.

\section{Mondo Sonoro}

http://www.mondosonoro.com/ (2006-07-03)

Ofrece, a través de la Red, entrevistas, noticias musicales, buscador, agenda, cine, conciertos, etcétera.

\subsection{Unidades de información: centros de documentación, archivos y bibliotecas (tanto de ámbito público como privado)}

Centro de Documentación Musical de Andalucía http://www.cdmandalucia.com/ (2006-07-03)

Ubicado en Granada, es un organismo creado por la Consejería de Cultura de la Junta de Andalucía con el fin de recuperar, custodiar, catalogar, clasificar y difundir el material musical creado o relacionado con Andalucía en todas sus formas: partituras, grabaciones sonoras y audiovisuales, manuscritos... A través de su web 
podemos consultar los fondos del catálogo bibliográfico de la biblioteca y de los recursos musicales de Andalucía, en cuyo proceso técnico se ha utilizado la herramienta ABSYS. Se compone de más de 41000 materiales (monografías, publicaciones periódicas, música impresa, casetes, cilindros, cintas magnetofónicas, DATS, discos compactos, de vinilo, de cartón de pizarra, rollos de pianola y vídeos). Sus bases de datos se dedican a compositores y entidades musicales. El Centro de Documentación tiene digitalizadas las noticias de prensa musical desde 1997.

(N. del E.: posteriormente el URL cambió a http://www.juntadeandalucia.es/ cultura/centrodocumentacionmusical/).

William and Gayle Cook Music Library

http://www.music.indiana.edu/muslib.html (2006-07-03)

Depende de la biblioteca de la Universidad de Indiana y tiene numerosos enlaces.

Fonoteca de la Casa de América

http://www.casamerica.es/ (2006-07-03)

Situada en Madrid, está compuesta por videoteca, archivo de exposiciones, archivo de multimedia, biblioteca, archivo de cine y fonoteca. Esta conserva un variado repertorio de ritmos y tendencias musicales de países latinoamericanos, desde los más conocidos, como tangos, rancheras, boleros o salsa, hasta aquellos menos difundidos en los circuitos europeos. Contiene 8000 temas latinos en diferentes soportes.

Centro de Documentación de Música y Danza http://cdmyd.mcu.es/ (2006-07-03)

En el año 1978 apareció en el Boletín Oficial del Estado la creación de este centro, que se ubicó en el edificio del Teatro Real de Madrid. En 1985 se constituyó el Centro de Documentación Musical como parte integrante y apoyo a la Dirección General del INAEM, del entonces Ministerio de Cultura. En 1996 se incorporó la sección de Danza. Cambió entonces su denominación por la actual y pasó a depender de la Subdirección General de Música y Danza. Tiene como funciones principales recopilar, sistematizar y difundir la información relativa a estas dos manifestaciones artísticas. Sus principales secciones son biblioteca, fonoteca, hemeroteca, archivo de partituras, videoteca, archivo de programas y carteles, y archivo fotográfico de organología.

Biblioteca Virtual de Educación Musical (BIVEM) http://80.34.38.142/bivem/ (2006-07-03)

Es la primera biblioteca virtual en español especializada en recursos para la educación musical. Sus fondos están compuestos por más de 850 enlaces y otros documentos electrónicos. Cada una de las entradas ofrece datos referidos a título, autor y materia del recurso, además de un resumen de su contenido.

Scire. 13 : 2 (jul.-dic. 2007) 167-184. ISSN 1135-3716. 
Mediateca del Institut de Recherche

et Coordination Acoustique/Musique (IRCAM)

http://mediatheque.ircam.fr/ (2006-07-03)

Su sede está París, en el Centre Georges Pompidou. A través de Internet, ofrece recursos y servicios on-line (buscador, bases de datos, consultas a los catálogos, enlaces a bibliotecas y librerías, etcétera), e incluso la posibilidad de escuchar discos en la Red.

Fonoteca Municipal de Lisboa

http://www.cm-lisboa.pt/fonoteca/ (2006-07-03)

Pertenece a la Cámara Municipal de Lisboa y es miembro de la Asociación Internacional de Bibliotecas Musicales. Su principal misión es servir de apoyo a los profesionales de la música en general y de Portugal en particular. Desde su portal nos informamos sobre su catálogo documental, publicaciones, funciones, etcétera.

(N. del E.: posteriormente el URL cambió a http://fonoteca.cm-lisboa.pt).

\subsection{Centros encargados de recopilar las producciones nacionales de música (generalmente adscritos a bibliotecas nacionales)}

\section{Francia}

La Biblioteca Nacional de Francia (http://www.bnf.fr) conserva cerca de 2 millones de documentos musicales, algunos de los cuales se pueden solicitar en caso de que no estén disponibles en los comercios. Además, en Francia también hay una biblioteca-museo dedicada a la ópera.

Suiza

Desde la web de la Fonoteca Nacional (http://www.fonoteca.ch) se puede encontrar información discográfica y consultar bases de datos, catálogos, colecciones, etcétera.

Italia

El Istituto Centrale per il Catalogo Unico delle Biblioteche Italiane e per le Informazioni Bibliografiche (ICCU) forma parte del Servizio Bibliotecario Nazionale (con más de 250000 registros de partituras manuscritas e impresas). Desde la página web (http://www.sbn.it/) podemos consultar otros organismos italianos que son promovidos por los Services on Music in Italy.

\section{Canadá}

Desde el portal principal de su Biblioteca Nacional accedemos al Index des Périodiques de Musique Canadiens (http://www.collectionscanada.ca/collection/ index-f.html) y podemos entrar a 25000 registros de 500 publicaciones musicales.

Scire. 13 : 2 (jul.-dic. 2007) 167-184. ISSN 1135-3716. 


\section{Brasil}

Accediendo a la Divisão de Música e Arquivo Sonoro de su Biblioteca Nacional (http://www.bn.br/site/pages/bibliotecaDigital/bibsemfronteiras/musica/index.html) podemos localizar sus fondos documentales. Tiene 220000 referencias, entre las que destacamos las colecciones de la Real Biblioteca y de Thereza Cristina Maria; conserva primeras ediciones de Haydn, Mozart, Beethoven y otros compositores de los siglos XVIII y XIX. Ofrece asimismo otros servicios virtuales.

\section{Estados Unidos}

La Library of Congress, en su sección Performing Arts Reading Room (http://lcweb.loc.gov/rr/perform/), conserva 12 millones de artículos dedicados a la música, además de partituras, bibliografía sobre músicos, 500 colecciones de música, teatro y danza, recursos digitales, publicaciones, acceso a los catálogos, bases de datos, guías, etcétera. Su misión principal es la difusión y conservación de sus fondos musicales.

\section{Reino Unido}

Las Music Collections de la British Library (http://www.bl.uk) ofrecen información sobre sus fondos mediante la consulta en los catálogos, el archivo de compositores y una amplia bibliografía especializada en música. Permiten búsquedas por estilos, artistas, etcétera.

\section{España}

Dependiente de la Biblioteca Nacional de España encontramos la sección de Grabaciones Sonoras (http://www.bne.es/esp/colecciones/grabaciones.htm). Contiene, por una parte, los llamados "fondos modernos", que proceden en su mayoría de los recibidos por depósito legal, y los fondos en soportes más antiguos, que ingresan fundamentalmente mediante adquisición. La colección de fondo antiguo es un fiel reflejo de la historia de la edición fonográfica española, y comprende desde los primeros soportes (discos de aristón, cilindros de cera, rollos de pianola) hasta los más modernos. La colección actual de rollos de pianola consta de aproximadamente 5000 volúmenes. Los fondos en discos de pizarra surgieron al establecerse el depósito legal para obras fonográficas en 1938. La colección inicial continúa incrementándose; actualmente supera los 12000 discos. Los discos de vinilo, cartuchos, casetes y discos compactos llegan también a la biblioteca vía depósito legal. Finalizado 2003, el total de documentos sonoros en diversos soportes depositados en este servicio superaban los 510000 ejemplares. Desde su web también podemos consultar el catálogo de esta sección de grabaciones sonoras.

\subsection{Universidades españolas donde se imparten estudios superiores de música}

La licenciatura en Historia y Ciencias de la Música tiene como finalidad capacitar al alumno para realizar tareas profesionales en los siguientes ámbitos: 
a) docencia: universitaria, bachillerato, enseñanza secundaria obligatoria, conservatorios $\ldots ; b$ ) documentación musical: fuentes documentales musicales, fuentes sonoras, organología, iconografía y archivos particulares; $c$ ) sonido: grabación, conservación y restauración sonora, producción musical, medios audiovisuales, edición musical; $d$ ) crítica musical en repertorios académicos, folclóricos y populares; $e$ ) gestión musical: asesoramiento y dirección de la programación de ciclos musicales, talleres de animación y difusión musical, iniciativas etnográficas y festivales folclóricos, tanto en instituciones públicas (ayuntamientos, diputaciones...) como en empresas privadas; $f$ ) asesoramiento en la restauración del patrimonio musical (instrumentos y material).

Esta titulación está integrada en los planes de estudios de la Universidad Complutense de Madrid (http://www.ucm.es/info/ucmp/pags.php?tp=Estudios\% 20Universitarios $\% 20 \mathrm{de} \% 20$ solo $\% 20$ Segundo $\% 20 \mathrm{Ciclo} \& \mathrm{a}=$ estudios $\& \mathrm{~d}=$ muestratitulacion.php\&idt=68), la Universidad de Granada (http://www.ugr.es/ mpaz/webpage8.htm), la Universidad de Valladolid (http://www.uva.es/consultas/ asignaturas.php?ano_academico=0809\&codigo_plan=289), la Universidad de Oviedo (http://directo.uniovi.es/catalogo/DetallePlan.asp?plan=HISTMUS2), la Universidad de Salamanca (http://www.usal.es/web-usal/Estudios/titulaciones/Historia ciencias_musica(2_ciclo).pdf) y la Universidad de La Rioja (http://campusvirtual. unirioja.es/titulaciones/musica/index.html), que ofrece la licenciatura on-line.

\subsection{Derecho de autor y propiedad intelectual}

El derecho de autor consiste en las facultades morales y de explotación que se le reconocen al autor de una obra original. Se considera autor a la persona natural que crea una obra literaria, artística o científica. En determinados casos las personas jurídicas también pueden ser titulares de derecho de autor, aunque nunca se les puede considerar creadores.

La propiedad intelectual en su concepto más amplio, que abarca tanto el derecho de autor (las facultades que se reconocen al autor de una obra) como los llamados "derechos conexos al derecho de autor" (que son facultades previstas a favor de otros agentes que intervienen en la creación de una obra, como los artistas, intérpretes o ejecutantes, los productores de fonogramas y de grabaciones audiovisuales, las entidades de radiodifusión, etcétera).

Sociedad de General de Autores y Editores ( $S G A E$ )

http://www.sgae.es/home/es/Home.html (2006-07-03)

Tiene delegaciones en varias comunidades autónomas y en el extranjero. Los autores o editores que pertenecen a la SGAE pueden declarar sus obras y percibir sus derechos. Sus obras han de ser audiovisuales, dramáticas o coreográficas (artes escénicas y musicales), o musicales. Desde su web podemos informarnos sobre reparto de derechos, reclamaciones, registro de obras, asesoramiento jurídico, etcétera.

Scire. 13 : 2 (jul.-dic. 2007) 167-184. ISSN 1135-3716. 
Centro Español de Derechos Reprográficos (CEDRO) http://www.cedro.org/inicio.asp (2006-07-03)

Tiene su sede en Madrid y cuenta con una delegación en Cataluña. Es una asociación sin ánimo de lucro de autores y editores de libros, revistas y otras publicaciones, editadas en cualquier medio y soporte, que se encarga de defender y gestionar de forma colectiva sus derechos de propiedad intelectual de tipo patrimonial (reproducción, transformación, comunicación pública y distribución). Fue autorizado para ello en 1988 por el Ministerio de Cultura, al amparo de la Ley de Propiedad Intelectual. En su página web podemos encontrar información sobre los tipos de derecho de autor, legislación y asociaciones de editores y autores, boletín informativo, dosieres de prensa, asesoramiento jurídico, buzón antipiratería, agenda, etcétera.

Asociación Española de Derecho de la Propiedad Intelectual (AEDPI) http://www.aedpi.com (2006-07-03)

Con sede en Madrid, se creó con el objetivo de promover, divulgar y salvaguardar los derechos que la Ley de Propiedad Intelectual confiere a sus titulares.

\section{Grupo Español de la Association Internationale}

pour la Protection de la Propiété Intellectuelle (AIPPI)

http://www.aippi.es (2006-07-03)

Representa en España los intereses de la AIPPI, asociación cuyo objetivo es promover la protección de la propiedad industrial e intelectual en todo el mundo.

Instituto de Derecho Industrial (IDIUS)

http://www.usc.es/idius (2006-07-03)

Este Instituto, integrado en la Universidad de Santiago de Compostela, tiene como objetivo principal promover, impulsar y desarrollar el conocimiento, la investigación y la difusión del derecho de la competencia, propiedad industrial y propiedad intelectual. Su web incluye un completo índice de textos legales.

Portal de la Universidad de Alicante

sobre propiedad industrial e intelectual y sociedad de la información http://www.uaipit.com/ (2006-07-03)

Fue presentado públicamente en junio de 2000, y nació con la intención de contener el mayor número posible de documentos agrupados en cinco categorías: protección de invenciones, protección de signos distintivos, protección del derecho de autor, sociedad de la información y aspectos generales. Incluye, además, interesante información sobre la Unión Europea y América Latina.

Scire. $13: 2$ (jul.-dic. 2007) 167-184. ISSN 1135-3716. 
Subdirección General de Propiedad Intelectual (Ministerio de Cultura) http://www.mcu.es/propint/index.jsp (2006-07-03)

La Subdirección General de Propiedad Intelectual ejerce las competencias del Ministerio de Cultura en esta materia, entre las que se encuentra la relación con las entidades de gestión colectiva de derechos.

(N. del E.: posteriormente el URL cambió a http://www.mcu.es/propiedadInt/ index.html).

Association Internationale pour la Protection de la Propriété Intellectuelle (AIPPI) http://www.aippi.org (2006-07-03)

Organismo independiente, con sede en Suiza, cuyo objetivo es promover la protección de la propiedad industrial e intelectual en todo el mundo.

Association Littéraire et Artistique Internationale (ALAI)

http://www.alai.org (2006-07-03)

Asociación creada en 1878 con el objetivo de que se convirtiera en un foro internacional para defender y promover los principios jurídicos que garantizan la protección internacional del derecho de autor.

Boletín de Derecho de Autor de la UNESCO

http://portal.unesco.org/culture/es/ev.php@URL_ID= 5130\&URL_DO=DO_TOPIC\&URL_SECTION=201.html (2006-07-03)

Acceso on-line al boletín sobre derecho de autor que edita la UNESCO.

Colección legislativa sobre la propiedad intelectual http://clea.wipo.int/ (2006-07-03)

Sitio web de la Organización Mundial de la Propiedad Intelectual (OMPI), donde se recogen todas las leyes relacionadas con la propiedad intelectual.

Intellectual Property Law Server

http://www.intelproplaw.com (2006-07-03)

Este servidor ofrece información sobre la legislación de propiedad intelectual, incluyendo patentes, marcas y derecho de autor.

International Federation of Reproduction Rights Organisations (IFRRO) http://www.ifrro.org (2006-07-03)

Fundada en 1988, esta federación agrupa a entidades de gestión colectiva de derechos reprográficos de todo el mundo y que trabaja para concienciar a la sociedad sobre la importancia de este tipo de entidades como mecanismos indispensables para una efectiva defensa de la propiedad intelectual de editores, escritores y otros titulares de derechos.

Scire. 13 : 2 (jul.-dic. 2007) 167-184. ISSN 1135-3716. 
International Intellectual Property Institute (IIPI) http://www.iipi.org (2006-07-03)

Instituto internacional creado en 1998 para desarrollar actividades relacionadas con la propiedad intelectual: publicaciones, seminarios, conferencias, etcétera.

Organización Mundial de la Propiedad Intelectual (OMPI) http://www.wipo.org (2006-07-03)

Organismo internacional cuyo objetivo es velar por la protección de los derechos de los creadores y los titulares de propiedad intelectual y lograr el reconocimiento y la remuneración que los autores, inventores y artistas deben percibir por la utilización de sus obras.

Organización Mundial del Comercio (OMC)

http://www.wto.org (2006-07-03)

Desde su página web se puede acceder a las leyes nacionales sobre derecho de autor y derechos conexos de los estados miembros de la UNESCO. Esta organización internacional se ocupa de las normas que rigen el comercio entre los países y elabora numerosos acuerdos, como el Acuerdo sobre los Aspectos de los Derechos de Propiedad Intelectual relacionados con el Comercio (Acuerdo sobre los ADPIC), que puede consultarse en este sitio web.

\section{UNESCO}

http://portal.unesco.org/culture/es/ev.php@URL_ID= 14076\&URL_DO=DO_TOPIC\&URL_SECTION=201.html (2006-07-03)

Colección de leyes nacionales sobre derecho de autor.

\section{Conclusiones}

La realización de este trabajo nos ha servido para demostrar, una vez más, cómo a través de Internet podemos acceder a numerosos recursos virtuales, en este caso dedicados a la música, ya que la red de redes acoge multitud de portales relacionados con la documentación musical.

Una vez llevado a cabo el proceso de búsqueda y recopilación del material, se seleccionó y analizó un variado abanico de recursos y fuentes de información electrónica, en concreto 65 , vinculados con la música de todos los géneros: centros de documentación, bibliotecas musicales, titulaciones universitarias, etcétera, ubicados en diferentes países. La mayoría de ellos ofrecen sus servicios y funciones online y brindan la posibilidad de participar en foros de debate o listas de discusión y de noticias.

Hemos conocido que las fuentes de información, generalmente, comparten varias tipologías: guías y directorios, catálogos y bases de datos, glosarios y tesau- 
ros... Lo mismo ocurre con las colecciones documentales de las unidades de información, que están formadas por monografías, publicaciones periódicas, música manuscrita e impresa, grabaciones sonoras - cilindros de cara, rollos de pianola, discos de metal, cartón, pizarra y vinilo, CD, carretes de cintas, casetes-, material visual y archivos de ordenador.

Casi todos los portales que tienen un buscador ofrecen la posibilidad de realizar búsquedas por géneros, artistas o álbumes.

Por no extendernos demasiado en este estudio, hemos reseñado brevemente la información contenida en los portales, en algunos casos contrastada con responsables de los centros, aunque puede ser ampliada en las direcciones webs que aportamos.

Brindamos los resultados obtenidos como base para futuros estudios. Además, pueden servir de guía para especialistas en documentación sonora, estudiantes de conservatorios de música, investigadores o musicólogos, centros de enseñanza, músicos, organizaciones y entidades relacionadas con el mundo de la música y la danza.

Sin duda, la era digital aporta la solución a muchos problemas de obtención de información y consulta de documentos, ya que facilita el acceso inmediato a bases de datos, catálogos de bibliotecas y un largo etcétera. Por ello, las autopistas de la información han supuesto un cambio importante para poder llevar a cabo este trabajo.

\section{Referencias}

Iglesias Martínez, Nieves (1992). Música: biblioteca de referencia. Madrid: Centro de Coordinación Bibliotecaria, 1992.

Iglesias Martínez, Nieves (1996). La edición musical en España. Madrid: Arco Libros, 1996.

Merlo Vega, José Antonio (1998). Música, webmaster: fuentes de información en línea para bibliotecas musicales. // Educación y Bibliotecas. 10:93 (1998) 60-65.

Miranda Regojo, Fátima (1990). La fonoteca. Madrid: Fundación Germán Sánchez Ruipérez, 1990, 2. ${ }^{\mathrm{a}}$ ed.

Parra Pérez, Águeda (1996). Música en Internet. Madrid: Anaya Multimedia, 1996.

Plaza-Navas, Miquel Àngel; Cuende, Maite (1999). La documentación musical en España ante el reto de las nuevas tecnologías: presente y... ¿futuro? // Jadoc'99: 2. ${ }^{\text {as }}$ Jornadas Andaluzas de Documentación : Nuevos mercados, nuevos usuarios. Granada: Asociación Andaluza de Documentalistas, 1999. 403-424.

Recursos musicales en España (1994). Madrid: Ministerio de Cultura, 1994.

Torres Mulas, Jacinto (1991). La documentación musical: luces y sombras. // Boletín de la Fundación Juan March. 212 (sept. 1991) 3-18.

Wallon, Simone (1984). La documentation musicologique. París: Beauchesne, 1984.

Scire. $13: 2$ (jul.-dic. 2007) 167-184. ISSN 1135-3716. 


\section{Anexo}

Bibliotecas especializadas en música, bibliotecas virtuales de partituras, institutos y centros de música, compañías discográficas, conservatorios de música, universidades, fundaciones, librerías y tiendas especializadas, orquestas y salas de conciertos

http://www.libraries.iub.edu/index.php?pageId=90

http://www.lib.duke.edu/dw3/SPT—BrowseResources.php?ParentId=177

http://hcl.harvard.edu/libraries/\#loebmusic

http://gopher.nypl.org/research/lpa/mus/mus.html

http://www.musiclibraryassoc.org/

http://www.partituras.com/index.asp

http://www.cim.edu/index.php

http://www.gruposyconciertos.com/discograficas.htm

http://www.musikene.net/

http://www.educa.madrid.org/web/csm.realconservatorio.madrid/

http://www.consuperiorsal.com/

http://www.consup-ib.com/

http://www.conservatori-liceu.es/wcast/conservatori/centres.htm

http://www.conservatoriovitoria.com/index.shtml

http://www.conservatoriosuperiorgranada.com/

http://www.conserv-sup-malaga.com/

http://www.csmmurcia.com/

http://www.pnte.cfnavarra.es/cmpablos/consernew/2quienes/hist.htm

$\mathrm{http}: / /$ web.educastur.princast.es/cons/conovied/

http://www.conservatori.net/

http://www.conservatori.net/

http://www.classical.net/music/links/musiclib.html

http://www.esmuc.net/

http://www.pnte.cfnavarra.es/ cmpablos/

http://www.unirioja.es/cvirtual/htmls/musica.html

http://www.uniovi.es/Vicerrectorados/Estudiantes/Estudios/Carreras/

LICENCIADOENHISTORIAYCCDELAMUSICA_2CICLO.html

http://www.ugr.es/ mpaz/webpage6.htm

http://www.uva.es/consultas/titulacion.php?menu=plan\&codigo_mec=

30800000000 \& centro $=0 \&$ campus $=0$

http://www.fundacionalbeniz.com/

http://www.libreriasespecializadas.com/musica.php

http://www.elargonauta.com/

http://www.casadellibro.com/

Scire. $13: 2$ (jul.-dic. 2007) 167-184. ISSN 1135-3716. 
http://home.palaumusica.org/

http://www.amazon.com/exec/obidos/tg/browse/-/538588/104-5646291-3069564

http://www.cduniverse.com/default.asp?style=music $\&$ cart $=149516606 \& \mathrm{frm}=$

http://www.amazon.com/exec/obidos/tg/browse/-/3023481/ref\%3Dtab\%5Fm\%5Fgw\%5Fl/

104-5646291-3069564

http://www.fnac.es/dsp/;jsessionid=aaaqAp0ehRKlfbqoQ_m?servlet=tree.

HomeTreeServlet $\&$ Code $1=2609895518 \&$ Code $2=9 \&$ tree $=1 \&$ catID $=-1 \&$ scType $=0$

http://www.elcorteingles.es/tiendas_e/cda/Musica/Home/0,4339,ECI,FF.html

http://www.discoweb.com/es/music/home.aspx_.html

http://www.tipo.es/

http://www.auditorionacional.mcu.es/

http://balletnacional.mcu.es/

http://cndanza.mcu.es/

http://www.liceubarcelona.com/teatreliceu.asp

http://orquestanacional.mcu.es/

http://www.rtve.es/oficial/orquesta_coro/index.html

http://teatrodelazarzuela.mcu.es/

http://www.teatro-real.com/

\section{Fabricantes de instrumentos musicales}

http://www.arrakis.es/ josugp/lutpercusion.htm

http://www.arhpa.com/

\section{Música clásica en Internet}

http://www.melomanos.com/

http://www.partituras.com/clasica/index.asp

http://www.catclassics.com/asp/index.asp

http://www.classical.net/

Otras webs dedicadas a ópera y a jazz son:

http://opera.stanford.edu/main.html

http://allaboutjazz.com

\section{Recursos para crear una web}

http://www.iaupa.com/Recursos_webmaster/

http://www.webestilo.com/recursos/recursos.php3

http://club.host.sk/webmaster1.html

\section{Software}

http://www.maz-sound.com/

http://www.finalemusic.com/

http://cctr.umkc.edu/userx/bhugh/musicold.html

Scire. 13 : 2 (jul.-dic. 2007) 167-184. ISSN 1135-3716. 
184 María F. Sánchez Hernández

http://www.musicsoft.es/

http://www.hitsquad.com/smm/

\section{Revistas}

http://www.omm.de/index.htm

http://www.rollingstone.com/home

http://www.sun.rhbnc.ac.uk/Music/Links/journals.html

http://www.billboard.com/bbcom/index.jsp 